La política de formación docente desde el proyecto de modernización educativa:

Elementos de debate

The policy of teacher training from the educational modernization

project: Elements for debate

Edith Lima Báez

Universidad la Salle Pachuca

Nota sobre el autor

Licenciada en Pedagogía por la Facultad de Filosofía y Letras de la UNAM, candidata a Maestra en Educación por la Universidad Pedagógica Nacional - Hidalgo. Docente de la Escuela de Ciencias Humanas de la Universidad La Salle de Pachuca y profesora asociada "A" por la UPNHidalgo.tutankamonplus@yahoo.com.mx

Esta investigación fue financiada con recursos del autor.

Remita cualquier duda sobre este artículo al siguiente correo electrónico: tutankamonplus@yahoo.com.mx

Recibido:1/1/2006

Aceptado:1/1/2006

Derechos de autor (C) 2006 Universidad La Salle Pachuca - Todos los derechos reservados.

Condiciones de Uso: La reproducción total o parcial de la obra en soporte electrónico queda prohibida sin la autorización de la Universidad la Salle Pachuca. 


\title{
La política de formación docente desde el proyecto de modernización educativa: Elementos de debate
}

Resumen

Edith Lima Báez ${ }^{\star}$

La modernización educativa en México ha generado políticas en torno a la formación docente de profesores de educación básica. Tales políticas de formación se constituyen como posibles acciones que resuelvan los problemas históricos de la profesión docente y los rezagos educativos existentes en el Sistema Educativo Nacional. La revisión analítica de tales propuestas ayudará a caracterizar la formación docente en los albores del siglo XXI.

\begin{abstract}
The educative modernization in México has generated political around the educational formation of professors in basica education. Such policies of formation are constituted like possible actions that solve the historical problems of the educational profession and the existing educative retags in the National Educative System. The analytical revision of such proposals will help to characterize the educational formation in the century XXI.
\end{abstract}

\section{A manera de introducción.}

La política es una praxis que se constituye en el plano de lo público a través de las relaciones entre los sujetos, de las negociaciones y acuerdos establecidos entre ellos mediados por intenciones que tienen como finalidad no sólo la representación social. Por lo que la política como hacer común, como hacer de todos, no es nunca privada, es multirrelacional.

Por un lado las políticas públicas o políticas estatales son "un conjunto de acciones y omisiones que manifiestan una determinada modalidad de intervención del Estado con relación a una cuestión ${ }^{[1]}$ que concita la atención, interés o movilización de otros actores en la sociedad civil” (OZLAK, 1976:16), es decir, son directrices que demarcan actividades y posibles soluciones a necesidades y 
problemáticas propias de cada esfera social, por ejemplo, en lo económico y en lo cultural, pero desde el ámbito estatal. Por otro están las costumbres, dicho en propiedad, el habitus de los sujetos.

Con respecto al primer punto, el campo de la educación también ha sido objeto de la implementación y aplicación de políticas públicas. Los sistemas educativos adoptan políticas para impulsar propuestas de cambio. Las políticas educativas configuran las decisiones que deben guiar el ejercicio del Estado desde el Sistema Educativo, por lo que los planteamientos de dichas políticas pretenden concretarse en acciones que coadyuven al logro de los propósitos de la educación. De esta forma los sistemas educativos tienen como objetivo satisfacer los requerimientos educativos de la sociedad a la que sirven.

De igual forma toda política educativa conlleva una filosofía, una concepción de hombre, sociedad, ciudadanía y educación. Dichos referentes son puntos nodales en las acciones educativas, por lo que se requiere tenerlas presentes. Tales aspectos se configuran a partir del espacio y tiempo histórico en las que se concretan. Así las políticas educativas se plantean como iniciativas, respuestas explícitas o implícitas a la educación, pero siempre en un marco histórico y en un contexto determinado. De ahí que habría que reconocer qué, cómo y cuáles son los procesos históricos que demarcan, construyen y posibilitan la implementación e impacto de las políticas educativas.

Particularmente la educación en México se encuentra desde hace varios años en un momento de reformulación. Dicho sentido reformista responde, por un lado, a programas internacionales, pero también, a la singularidad del sistema educativo mexicano, es decir, a las prácticas políticas arraigadas (NORIEGA, 2000).

El Nuevo Modelo de Desarrollo Educativo en México, se ha venido formulando desde la década de los 70's, pero se concreta en la década de los 90's y se le reconoce como el proyecto de modernización educativa. 
En esta perspectiva y para fines de este artículo, importa no sólo reconocer las acciones generadas en torno a la formación de profesores de educación básica de fines de siglo XX y principios del siglo XXI en México, sino también los elementos económicos y políticos que las impulsan y dan sustento, con la finalidad de caracterizar a la formación docente.

\section{La formación docente en el proyecto de modernización educativa.}

Cuando escuchamos hablar de modernización educativa, imaginamos procesos de reforma, de cambio, renovación e innovación. De ahí que pensemos que es un proyecto fundamental para el sistema educativo. Sin embargo, es importante reconocer que detrás de todo proceso de modernización se encuentran los referentes de la modernidad como metarrelatos. ${ }^{[2]}$ La modernidad propone que toda concepción, proceso y actividad debe girar en torno a la razón del ser humano, por ello los referentes centrales de ésta son la búsqueda del progreso y el desarrollo basados en la actividad científica y tecnológica. De ahí que la modernización tienda al progreso, a plantear los ejes de desarrollo social y económico. La Modernidadcomo época histórica se conforma de una red de modernizaciones. Este es un primer referente fundamental del proyecto de modernización educativa en México.

Un segundo referente que sustenta a tal proyecto es la postura económica de principios del siglo XXI: la globalización, que tiene como fundamento filosófico al neoliberalismo. ${ }^{[3]}$ La globalización tiene un impulso después de la caída del muro de Berlín; pretende unificar al mundo a partir de las relaciones económicas, políticas y culturales. Con tal movimiento se empiezan a desdibujar las fronteras entre los países "La tendencia que se observa a nivel mundial es la progresiva integración de 'bloques de naciones' entre los que se encuentran el asiático, la comunidad europea, Norteamérica, el mundo Árabe y América Latina" (MENDOZA, 1995: 103). Sin embargo, la globalización no produce unificación cultural, social y política, sino una nueva lógica de relaciones, dependencias y formas de vida (BECK, 1988).

Particularmente en México, el ingreso de las tendencias neoliberales y de globalización inició en el sexenio de Miguel de la Madrid, pero se consolida en los tres sexenios posteriores. Se privatizó la banca, se vendieron la mayoría de las 
empresas paraestatales a sectores privados y entre otras cosas, se generaron relaciones económicas entre países del primer mundo con nuestro país a través de Tratados de Libre Comercio. Este análisis se ve reflejado en el ámbito económico, social y político de México, pero de manera particular en el sistema educativo. A partir de la escolarización y formación de profesores se pretenden consolidar los nuevos valores sociales de eficacia, eficiencia, pertinencia, equidad y calidad. De ahí que si se quiere formar a un nuevo ciudadano vinculado a los preceptos anteriores, se requiere por lo tanto de nuevas propuestas de formación de profesores, sobre todo de educación básica pues ellos son los mediadores entre las reformas del Estado y la sociedad representada por padres, niños y jóvenes que asisten a las escuelas.

En este sentido, el proyecto de modernización educativa en México se divide en dos periodos: aparece en 1970 durante el sexenio echeverrista, continúa en los dos siguientes sexenios pero se redefine en 1988 con Carlos Salinas de Gortari. En la actualidad el proyecto sigue vigente. Carlos Ornelas (2000) define a la primera etapa como "los albores de la modernización" mientras que la segunda es la consolidación y transición del proyecto modernizador. Se distinguen tres grandes hechos significativos en este proyecto: "El impulso a la universalización de la enseñanza primaria (Educación para Todos y Primaria para todos los niños) de 1976 a 1982 y los procesos de desconcentración y descentralización que culminan en la "federalización" de 1992; a estas características habría que añadir el énfasis en la eficiencia y la calidad, observable ya desde la década de los ochenta" (LATAPÍ, 1998: 32).

La profesión docente vivió dos momentos importantes durante el proyecto modernizador de los 70 y 80: por un lado se impulsó la formación inicial y permanente, se estabilizó la matrícula en las escuelas normales, se alcanzó un porcentaje considerable de profesores titulados, pero por otro lado, se inició una crisis en la profesión (ARNAUT, 1996).

La crisis se generó debido a las condiciones laborales y salariales de los profesores, así como por las confrontaciones entre sectores magisteriales y el Sindicato Nacional de Trabajadores de la Educación(SNTE), pero también por la desvalorización social de la profesión docente. 
A partir de esta crisis, los docentes iniciaron una serie de movilizaciones al inicio del sexenio de Carlos Salinas de Gortari (1988-1994) que terminaría con la destitución del dirigente del SNTE por más de 15 años: Jongitud Barrios.

Con estos antecedentes nacionales y con el impulso de la globalización en el ámbito económico a nivel internacional, se inició la transición del proyecto de modernización educativa, ya que se planteó una reforma del sistema educativo nacional con un nuevo modelo de desarrollo: el neoliberalismo. En 1989 se presentó a nivel nacional el documento rector de esta reforma: El Programa para la Modernización Educativa, que mostraba el diagnóstico del sistema educativo desde educación inicial hasta el desarrollo científico y tecnológico del país (PODER EJECUTIVO FEDERAL, 1989).

Para el caso de la educación básica y con el propósito de resolver el conflicto existente con los profesores, el gobierno federal negoció junto con el sindicato una serie de acciones como el aumento al salario y la creación de carrera magisterial. Los logros alcanzados a partir de esta negociación se vieron reflejados en el Acuerdo Nacional para la Modernización de la Educación Básica (ANMEB) de 1992 y en la Ley Generalde Educación (1993) que fueron el parte aguas para las modificaciones en el subsistema de educación básica y normal.

El Acuerdo Nacional para la Modernización de la Educación Básica, consideró una serie de propósitos para la reorganización del sistema educativo. El primero de ellos remitió a la consolidación de un Nuevo Federalismo. Éste incluyó la decisión de transferir a los gobiernos estatales los servicios de educación básica y normal en términos de bienes patrimoniales, recursos presupuestales, infraestructura y equipo, recursos humanos que a partir de la firma del ANMEB quedaron bajo la responsabilidad de las Entidades Federativas. El Gobierno Federal a través de la SEP se encargó de elaborar planes y programas de estudio, de la formación de profesores y la elaboración de libros de texto gratuitos. Se promovió la participación social a través de los Consejos de Participación escolar, municipal, estatal y nacional, con la finalidad de que la sociedad se inmiscuyera en los problemas educativos.

Este proyecto Modernizador ha repercutido, como ya se mencionó, en la profesión de los docentes de educación básica y en los procesos de formación de estos últimos. Uno de los propósitos de la reforma modernizadora fue la revaloración 
social de magisterio, así como la definición de lineamientos para la formación y actualización de los docentes y la generación de una nueva conciencia profesional del maestro, es decir, se buscó "El compromiso de un salario profesional para que los educadores de los planteles públicos alcancen un nivel de vida decoroso para su familia; el establecimiento de mecanismos que propicien la permanencia de los maestros frente a grupo y la posibilidad para éstos de ir obteniendo mejores condiciones y mayor reconocimiento social; y, el otorgamiento de distinciones, estímulos y recompensas a los educadores que destaquen en el ejercicio de su profesión" (NORIEGA, 2000: 233).

Así, la revaloración social del docente implicó la formación y actualización de éste, por lo que se creó el Programa Nacional para la Actualización Permanente de los Maestros de Educación Básica en Servicio (PRONAP) quien coordina los centros de maestros, los cuales son espacios para el estudio individual y grupal de profesores en servicio, donde se imparten cursos que apoyen su práctica docente. A partir de 1992 se crearon 500 Centros que cuentan con una biblioteca básica de 2000 títulos, instalaciones para la recepción, grabación y reproducción de audio y video, además de contar con equipos docentes que asesoran a los profesores considerando ciertos paquetes didácticos (IBARROLA, 1998: 261-262). La hipótesis parece ser que con "Ilenar" a la escuela de tecnología moderna se superaría como por arte de magia la formación docente, cuestión bastante difícil. Para 1997, dentro del marco de la Modernización Educativa se modificaron los planes de estudio de las Normales (preescolar, primaria y secundaria). Con estas reformas, se pretendió formar a un maestro que investigue (igual que en el plan de normales de 1984) y que adecue los contenidos nacionales al espacio regional en que trabaja. No obstante estos planes carecen de asignaturas vinculadas a la didáctica y a la pedagogía, por lo que al parecer se pretende dar un giro en la formación docente.

En el sexenio foxista, la formación docente continúa en las lógicas de finales de siglo XX, los nuevos planes de estudio de la educación preescolar y secundaria, han llevado a los profesores en servicio a actualizarse y capacitarse en los modelos de competencia y valores vinculados a la cultura de la legalidad, respectivamente. 


\section{La caracterización de la formación docente, elementos de debate.}

Con los elementos de análisis vertidos en párrafos anteriores, en torno a las acciones de formación docente en el proyecto de modernización educativa, se puede reconocer que el desarrollo y configuración de la formación de profesores de educación básica en los albores del siglo XXI es heterogénea. Encontramos que la formación gira institucionalmente entre las escuelas normales y aquellos espacios destinados a la actualización docente, por lo que podemos reconocer dos tipos de formación institucional: la formación inicial, prioridad de las escuelas normales y sus distintas modalidades (rurales, urbanas, privadas) y la formación permanente. Esta última presenta diferenciaciones, por un lado, se trata de una formación para maestros en servicio con estudios o titulo de normal y para aquellos que requieren de la nivelación pedagógica debido a las reformas de planes de estudio tanto en las normales como en los diferentes niveles educativos. Pero por otro lado, se han habilitado a profesores que no son normalistas para las actividades de enseñanza en las escuelas.

Asimismo, se puede plantear que la formación docente es diferenciada de acuerdo a la zona de influencia y a la población a la que se atiende, es decir, no es lo mismo una formación dirigida a profesores que desarrollan su práctica docente en zonas rurales ${ }^{[4]} \mathrm{O}$ urbanas, ni tampoco para los profesores que atienden a poblaciones indígenas, con necesidades educativas especiales o distribuidos en edades y etapas cronológicas (niños, adolescentes, adultos).

Por otra parte, la diferenciación por género está presente en la formación docente. Socialmente se manifiesta que la profesión docente está constituida mayoritariamente por mujeres, las normales de educadoras siguen vigentes y en la actualidad, algunas normales rurales que prestan el servicio de internado conservan la separación entre hombres y mujeres.

Otro de los elementos que marcan la diversificación de la formación docente, es la especialización en ciertas áreas de conocimiento sobre todo para aquellos que asisten a las normales superiores: ciencias naturales, ciencias sociales, español, matemáticas, o bien, a normales especializadas en educación física, música y, 
educación especial (audición y lenguaje, ciegos y débiles visuales, deficientes mentales, problemas de aprendizaje, menores infractores)

Por las condiciones laborales de los profesores, también podemos encontrar diferencias en la formación, la que reciben los instructores comunitarios es diferente de aquella que se otorga a los profesores de la educación regular, aunque los dos desarrollan una práctica docente, los primeros no tienen prestaciones de ningún tipo pues ofrecen un servicio social; estos son formados por el Consejo Nacional de Fomento Educativo (CONAFE), quien no sólo recibe apoyo federal sino también, apoyo económico de organismos internacionales, por lo que sus programas de formación, capacitación y actualización difieren en cuanto a enfoques pedagógicos y sociales. Los segundos son formados directamente por el Estado y la mayoría tiene seguridad social.

\section{A manera de cierre.}

A partir de los referentes anteriores, se puede reconocer que las acciones de la política de formación docente de educación básica, a fines de siglo XX y principios del siglo XXI en México, están sustentadas e impulsadas por las tendencias neoliberales y de globalización presentes a nivel mundial.

Así, la modernización educativa en México pretende, aunque no lo clarifica abiertamente, que la formación de profesores gire en torno a tales propuestas, lo que haría pensar que la profesión docente tiene la misión de formar a ciudadanos competentes en términos de saber hacer, pero individualizados, aunque contradictoriamente, vinculados al reconocimiento de derechos humanos y la diversidad. Las acciones de la política de formación de profesores pretenden conformar un tipo de profesional de la educación vinculado a las nuevas perspectivas mundiales.

Sin embargo, a quienes diseñan y ponen en acción las políticas educativas se les olvida que todo proceso de formación conlleva un antecedente histórico que no se puede desconocer, de ahí la importancia de caracterizar la formación docente en nuestro país, situación que necesariamente remite a procesos de análisis histórico. 
A pesar de las múltiples propuestas de la política de formación docente en México y sus intentos de operativización, se presenta un abismo entre el discurso de formación y las prácticas de enseñanza. La formación como discurso de cambio se vuelve un punto de desencuentro entre los profesores y la realidad educativa, pues no siempre satisface sus necesidades, ni se les considera para elaborar las directrices de la política educativa, por lo que los profesores de educación básica, continúan con prácticas y concepciones educativas arraigadas.

La política educativa de formación docente, es un discurso discordante frente a una realidad socialmente construida, frente a prácticas y concepciones de sujetos configuradas en contextos particulares.

No obstante, se requiere hacer uso de tales divergencias para resignificar la función de la escuela obligatoria y de la profesión docente. Para ello, es fundamental escuchar las voces de los implicados; son los sujetos inmersos en los procesos escolares los que tienen que hacer un ejercicio de reflexión y autocrítica con relación a la forma en cómo se asumen las políticas estatales, con el propósito de articular lo tradicional y lo nuevo, lo instituido y lo instituyente, los discursos y las prácticas.

\section{Referencias}

ABBAGNANO, Nicola (2004), Diccionario de Filosofía, México: Fondo de Cultura Económica.

ARNAUT, Alberto (1996), Historia de una profesión. Los maestros de educación primaria en México 1887-1994, México: Centro de Investigación y Docencia Económicas.

BECK, Ulrich (1988), Qué es la globalización. Falacias del globalismo, respuestas a la globalización, México: Paidós.

IBARROLA, María de (1998), "La formación de profesores de educación básica en el siglo XX" en Pablo Latapí (2004) (Coord.), Un siglo de educación en México. Tomo II, México: Fondo de Cultura Económica/CONACULTA.

LATAPÍ, Pablo (1998), "Un siglo de educación nacional: una sistematización" en Pablo Latapí (2003) (Coord.), Un siglo de educación en México. Tomo I, México: Fondo de Cultura Económica/CONACULTA. 
MENDOZA Rojas, Javier (1995), "La Universidad frente a las tendencias de la globalización" enEscenarios para la universidad contemporánea, México: CESUUNAM.

NORIEGA, Blanca (2000), "Modernización educativa mexicana y sus políticas de financiamiento" en Las reformas educativas y su financiamiento en el contexto de la globalización; el caso de México, 1982-1994, México: UPN-Plaza y Valdez.

ORNELAS, Carlos (2000), El sistema educativo mexicano. La transición de fin de siglo, México: Fondo de Cultura Económica/CIDE/NAFIN.

OZLAK, O y O'Donell, G. (1976), Estado y políticas estatales en América Latina, Buenos Aires: CEDES/ CLACSO.

PODER EJECUTIVO FEDERAL (1989), Programa Nacional para la Modernización Educativa 1989-1994, México: SEP.

\footnotetext{
[1La cuestión o cuestiones son necesidades y demandas sociales que se problematizan por ciertos grupos en el afán de resolverlas de manera estratégica y que están en condiciones de incluirse en la agenda de problemas socialmente vigentes, o bien, en la agenda de gobierno.

${ }^{[2]}$ El metarrelato es un término empleado por J.F. Lyotard para definir "las grandes síntesis teóricas por medio de las cuales la modernidad ha procurado ofrecer una legitimación filosófico-política del saber" Abbagnano, 2004:718) Entre los metarrelatos de la modernidad se encuentran el modelo iluminista y el modelo idealista. El primero plantea que el saber es legítimo en tanto favorece la emancipación y libertad de los pueblos, y en el segundo, el saber se constituye como un conocimiento desinteresado que el espíritu tiene de sí mismo, por lo que no busca ningún fin.

${ }^{[3]}$ EI neoliberalismo es una doctrina filosófico-económica que revaloriza al liberalismo del siglo XVIII y XIX. Plantea el libre intercambio comercial, la libre competencia reguladora de la producción y de los precios, exalta la iniciativa individual movida por el deseo de lucro y propone la no injerencia del Estado en la vida social y económica.

${ }^{[4]}$ No obstante se tendría que reconocer que el proyecto de normales rurales está, según el Estado, en decadencia. A partir de 1998, las escuelas normales rurales están desapareciendo. (Véase el caso de la Normal Rural "Luis Villarreal", en Hidalgo)
} 